\title{
Ein neues Verfahren zur Bestimmung des Feinheitsgrades der Weizenmehle.
}

\author{
Von \\ L. v. Liebermann and V. Andriska. \\ Mitteilung aus dem Hygienischen Institut der Universitä Budapest. \\ (Direktor: Prof. L. v. Li ebermann.)
}

[Eingegangen am 29. April 1911.]

Einleitung.

Von L. v. Liebermann.

Die Bestimmung des Kleiegehaltes der Weizenmehle, mithin die ihres Feinheitsgrades, geschieht bisher nach ibrer Farbe und zwar nach vereinbarten, in verschiedenen Jahren sogar nicht unerheblich wechselnden, an gewissen Zentralstellen deponierten Typen, mit denen die zu untersuchenden Mehle verglichen werden. Ohne solche Typen ist es unmöglich, den Feinheitsgrad - die Nummer - eines Mehles zu bestimmen. Für die Bestimmung des wirklichen Kleiegehaltes aber fehlt bisher jede exakte Grundlage, sodaß man darüber auch dann kaum etwas Sicheres aussagen kann, wenn man im Besitz der erwähnten Typen, für ein zu untersuchendes Mehl die augenblicklich, d. $h$. für ein bestimmtes Jahr geltende Nummer angegeben hat.

Es hat bekanntlich an Vorschlägen, diesem Übelstand abzuhelfen, nicht gefehlt (Bestimmung des Aschengehalts, der Rohfaser, des Stickstoffs, des Fettes), doch ist man immer wieder zur Farbe zurückgekehrt und so ist in Ermangelung von etwas Besserem immer noch das „Pekarisieren“ das gebräuchlichste Verfahren.

Einer freundlichen Aufforderung des Herrn Professors Lindet, Präsidenten der Internationalen Analysen-Kommission, in Paris folgend, habe auch ich mich mit dieser Frage befaßt.

Wenn man Weizenmehl in einem Reagensglase mit Chloroform schüttelt, wie bei der bekannten Probe auf mineralische Verunreinigungen, so bemerkt man, daß sich die Chloroformlösung je nach dem Fettgehalt des Mehles mehr oder weniger stark färbt, das Mehl selbst aber mit der darin enthaltenden Kleie nach einigem Stehen in die Höhe steigt und auf der Oberfläche der Chloroformlösung eine je nach dem Kleiegehalt mehr oder weniger bräunliche, bei den feinsten Mehlen fast weiße Scheibe bildet. Man kann auf diese Weise aus den verschiedenen Mehlsorten eine ganze Skala von weiß bis fast dunkelbraun herstellen und hat den Vorteil, das bei solchen Farbenvergleichen sonst häufig störende Fett entfernt zu haben und die Möglichkeit, schon mit freiem Auge, jedenfalls aber mit der Lupe, die Gegenwart sowohl mineralischer Verunreinigungen (am Boden des Reagensglases), als auch Verunreinigungen mit Unkrautsamen, deren feingemablene Partikelchen mit dem Mehl und der Kleie in die Höhe steigen, in der Scheibe zu erkennen.

Macht man sich nun Gemische aus feinstem, fast kleiefreiem Weizenmehle und feingemahlener, stärkefreier Weizenkleie nach bestimmten Gewichtsverhältnissen, so kann durch colorimetrischen Vergleich mit diesen Typen von bekanntem Kleiegehalt der Feinheitsgrad, d. i. der Kleiegehalt jeden Weizenmehles bestimmt werden. 
Dieses ist das Prinzip eines Verfahrens, das ich mit Herrn Dr. Andriska ausgearbeitet habe. Der naheliegende Gedanke, zur Herstellung der Mischungen reines Stârkemehl zu verwenden, ist nicht durchfübrbar, da in die Bildung der obenerwähnten gefärbten Scheiben auch andere Bestandteile der Mehle eingehen, die einerseits auf die Farbe, andererseits auf die gleichmäfige Vertellung der Kleie von Einflub sind.

\section{Das neue Verfahren.}

\section{Von L. v. Liebermann und V. Andriska.}

1. Man bereitet sich zunächst einen Vorrat feinsten, stärkefreien Kleiemehles auf folgende Weise: Feinste Weizenkleie wird mit Wasser so lange ausgekocht, bis die dekantierte Flüssigkeit klar ist. Hierauf wird der Rückstand auf dem Wasserbade getrocknet und die getrocknete Kleie in kleinen Portionen in einem Porzellanmörser möglichst fein zerrieben. Die Masse wird abermals mit immer neuen Portionen Wasser ausgekocht bis zum Verschwinden der Stärkereaktion mit Jodlösung oder doch so. lange, bis die Jodreaktion nur mehr sehr schwach geworden ist.

Die nun nahezu stärkefreie Kleie wird abermals auf dem Wasserbade getrocknet, nochmals fein zerrieben und durch Müllergaze (D ufour \& Co. No. 18, Fädenzahl 66 auf $1 \mathrm{~mm}$ ) gesiebt.

2. Feinster Weizengrieb, der fast kleiefrei ist ${ }^{1}$ ), wird in einer Porzellanschale aufs feinste zerrieben.

3. Aus 1 und 2 werden nun Mischungsn nach bestimmten Gewichtsverhältnissen hergestellt und innig verrieben. Mit diesen Mischungen von bekanntem Kleiegehalt werden die zu prüfenden Mehle verglichen.

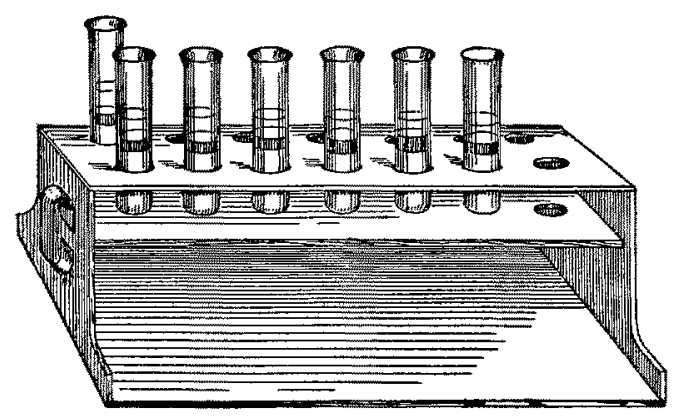

Bei der Herstellung unserer Skala haben wir uns aus praktischen Gründen an die Mehltypen der Budapester Börse gehalten, die einen Kleiegehalt von 0 bis $2 \%$ aufwiesen.

4. Hat man sich nun einen Vor. rat solcher Mischungen bergestellt, so nimmt man aus jeder Probe $1 \mathrm{~g}$ mit Hilfe eines Löffelchens, das, glatt gestrichen, gerade $1 \mathrm{~g} \mathrm{Mehl} \mathrm{fabt,} \mathrm{bringt}$ diese Proben in kurze, $10^{1 / 2}-11 \mathrm{~cm}$

lange, gleich weite, entsprechend bezeichnete Röhrchen, schüttelt jede mit $10 \mathrm{ccm}$ Chloroform tüchtig durch und stellt sie dann in ein weif angestrichenes Gestell vom nebenstehender Form.

Nach einstündigem Stehen haben sich über den mehr oder weniger gefärbten Chloroformlösungen $4-5 \mathrm{~mm}$ dicke Scbeiben gebildet, deren Färbung je nach dem Kleiengehalt der Mehlprobe verschieden ist. Man kann seitlich beobachten, aber

1) $\mathrm{Ob}$ ein Mehl als kleiefrei gelten kann, kann auch nach der Phloroglucin-Reaktion beurteilt werden. Schüttelt man $1 \mathrm{~g} \mathrm{Mehl} \mathrm{mit} 5 \mathrm{ccm}$ Chloreform, gibt dann 5 Tropfen einer mit $37 \%$ iger Salzsäure bereiteten Phloroglncinlösung zu und schüttelt abermals durch, so erscheint fast kleiefreies Mehl nach $1 / 4$-stündigem Stehen hell und rosafarben, während andere, minder reine Mehle, dunkler gefärbt sind and zwar mit einem Stich ins Gelbe oder Bräunliche. 
noch besser ist es, wenn man von obenher in die Röhrchen blickt. Es ist zweckmäßig, die Scheiben mit 1 ecm reinen Glycerins (spez. Gewicht 1,225-1,235) vorsichtig zu überschichten, indem man es an der Röhrchenwand hinabrinnen läßt. Eine solche Skala kann eine Woche lang oder noch länger stehen.

5. Nun nimmt man aus dem zu prüfenden Mehl ebenfalls $1 \mathrm{~g}$, verfährt genau auf die oben beschriebene Weise und vergleicht die Färbung der Mehlscheibe mit der nach 4. hergestellten Skala, indem man das Röhrchen in die verschiedenen Löcher der zweiten Reihe des Stativs steckt und zusieht, mit welcher Nummer der Skala die Probe am besten übereinstimmt.

Hat man sogenannte, griffige oder griebige Mehle zu untersuchen, so müssen diese vorher möglichst fein verrieben werden.

Wir lassen nun einige Bestimmungen folgen, die auch einen Anhaltspunkt dafür geben, in welchen Gewichtsverhältnissen die Vergleichsmischungen von bekanntem Kleiengehalt herzustellen sind.

Kleiegehalt der Typen der Bndapester Börse.

\begin{tabular}{|c|c|c|c|c|c|}
\hline \multicolumn{2}{|c|}{$\begin{array}{c}\text { I. Glattgemahlene } \\
\text { Mehle. }\end{array}$} & \multicolumn{2}{|c|}{ II. Griffige Mehle. } & \multicolumn{2}{|c|}{$\begin{array}{c}\text { III. Doppelgriffige } \\
\text { Mehle. }\end{array}$} \\
\hline $\begin{array}{l}\text { No. } \\
\text { des Mehles }\end{array}$ & Kleiegehalt & $\begin{array}{l}\text { No. } \\
\text { des Mehles }\end{array}$ & Kleiegebalt & $\begin{array}{c}\text { No. } \\
\text { des Mehles }\end{array}$ & Kleiegehalt \\
\hline 0 & 0 & 0 & $0,1 \%$ & 0 & $0.1 \%$ \\
\hline 1 & $0,1 \%$ & 1 & 0,2, & 1 & $0,2 "$ \\
\hline 2 & 0,2, & 2 & $0,5 n$ & 2 & 0,5, \\
\hline 3 & 0,4 & & & 3 & 0,8 \\
\hline 4 & 0,6, & & & 4 & 1,25 \\
\hline 5 & $0,9 "$ & & & & \\
\hline 6 & $1,25 "$ & & & & \\
\hline 7 & 1,5, & & & & \\
\hline $7^{1 / 2}$ & 2,0 & & & & \\
\hline
\end{tabular}

Die Untersuchung von Weizenmehl-Typen (1911) der Versuchsanstalt für Getreideverarbeitung in Berlin, Abteilung für Versuchsmüllerei, hat folgendes ergeben:

$\begin{array}{rcc}\text { Weizenmehl-Type } & \text { Ausbeute-Klasse } & \text { Kleiegehalt } \\ 0 \text { bis } 30 \% & \text { I } & 0 \\ \text { über } 30,70 " & \text { II } & 0,6 \% \\ " 70 " 75 " & \text { III } & 2,0, \\ 0 " 70 " & \text { IV } & 0,3 " \\ 0,75 ", & \text { V } & 0,5 "\end{array}$

Man kann sich natürlich die Vergleichsmischungen nach beliebigen Verhältnissen herstellen, doch dürften die obigen den gegebenen Verhältnissen entsprechen.

Die Ausbeuteklassen der Berliner Versuchsanstalt verhalten sich zu den Budapester Mehltypen, wenn man noch eine Mehlnummer $7^{3 / 4}$, die auch hergestellt wird, dazu nimmt, wie folgt:

$\begin{array}{cccc}\text { Budapester Nummern } & \text { Ausbente } & \text { Berliner Ausbeute-Klassen } & \text { Ausbeute } \\ 0 & 40 \% & \text { I } & 30 \% \\ 1,2,3,4,5,6,7 & 30 \% & \text { II } & 40 \% \\ 7^{\frac{1}{1} / 2,73 / 4} & 5 " & \text { III } & 5 \% \\ 0,1,2.3,4,5,6,7 & 70 " & \text { IV } & 70 " \\ 0-7^{3} / 4 & 75 " & \text { V } & 75,\end{array}$


Wir verdanken diese Aufstellung der Freundlichkeit des technischen Leiters der Pester Walzmühlengesellschaft, Herrn Dr. Emil Varsányi, der die Güte hatte, uns auch sonst durch Beschaffung einwandfreien Untersuchungsmaterials zu unterstützen.

Wir glauben, daß man, im Besitze dieser einfachen, von uns vielfach geprüften, hinreichend genauen Methode, nun recht wohl imstande ist, den Kleiegehalt der Weizenmehle zu bestimmen, mithin aber auch den Verkehr mit Weizenmehl auf die exakte Basis eines quantitativ bestimmten Kleiegehaltes zu stellen.

\section{Apparat zur Bestimmung des Schmelzpunktes von Fetten.} Von.

\section{L. v. Liebermann.}

Mitteilung aus dem Hygienischen Institut der Universitä Budapest.

[Eingegangen am 29. April 1911.]

In die U-förmig gebogene Röhre (s. Abbildung) füllt man etwas Quecksilber und darüber in den einen Schenkel der Röhre das vorher geschmolzene und klar

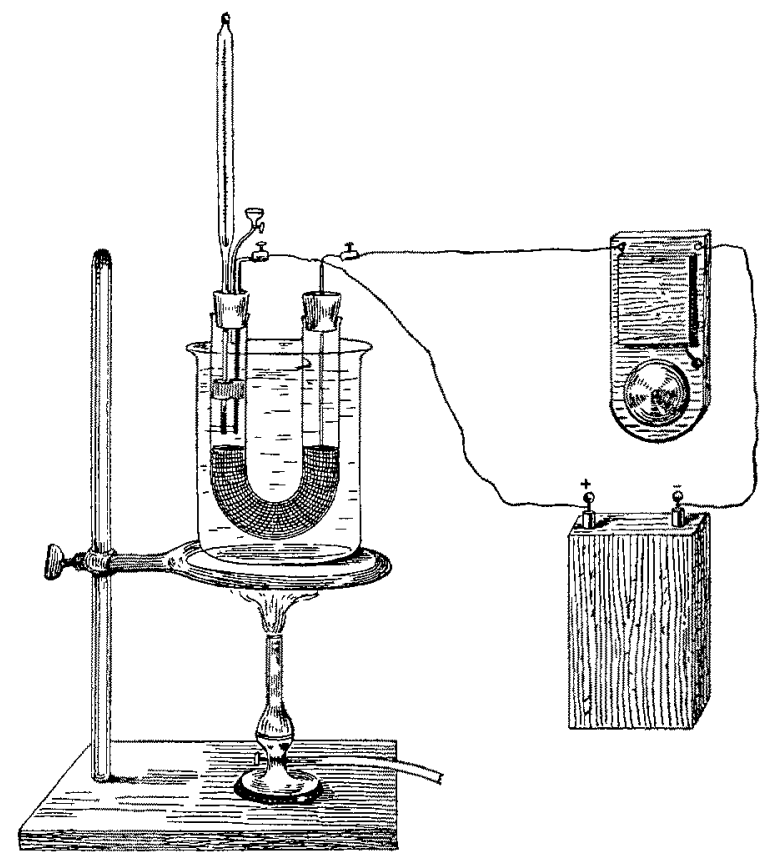

filtrierte Fett. Dann wird ein dreifach durchbohrter Stöpsel aufgesetzt; durch die eine Bohrung wird das Thermometer eingeführt, durch die andere ganz enge ein genügend dicker Platindraht. Die Kugel des Thermometers und das Ende des Platindrahts sollen bis in die Mitte der geschmolzenen Fettschichte reichen. Hierauf läßt man das Fett vollständig erstarren. Dies erfordert bekanntlich längere Zeit; nötigenfalls ist Kühlung mit Eis anzuwenden.

Ist dies geschehen, so stellt man die Röhre senkrecht in ein mit Wasser gefülltes Becherglas. und befestigt sie mit einer Klemme des Stativs.

Nun läft man durch einen kleinen Trichter, den man durch das dritte Bohrloch fübrt, etwas Quecksilber über die erstarrte Fettschicht fließen, etwa halb so hoch wie die Fettschicht selbst, steckt auch in den anderen Schenkel der Röhre einen Platindraht, verbindet beide Drähte mit einem Läutewerk und erwärmt das Wasserbad. Sobald das Fett zu schmelzen beginnt, fällt die obere Quecksilberschicht herunter, der Kontakt wird hergestellt und das Läutewerk tritt in Tätigkeit. Man 